Disclosure of Interests: Laurette van Boheemen: None declared, S.A. Turk: None declared, M.H. van Beers - Tas: None declared, W.H. Bos Grant/research support from: abbvie, sanofi, roche, celgene, ucb, novartis, Speakers bureau: abbvie, Sanofi, eli lilly, Diane Marsman: None declared, E.N. Griep: None declared, M. Starmans: None declared, C.D. Popa: None declared, A.M. van Sijl: None declared, Maarten Boers: None declared, Michael Nurmohamed Grant/ research support from: Not related to this research, Consultant of: Not related to this research, Speakers bureau: Not related to this research, Dirkjan van Schaardenburg: None declared

DOI: 10.1136/annrheumdis-2020-eular.2805

\section{AB0231 \\ RHEUMATOID ARTHRITIS PATIENTS TREATED WITH ABATACEPT AT A COMMUNITY RHEUMATOLOGY CLINIC AND WHO ARE POSITIVE FOR ANTICYCLIC CITRULLINATED PEPTIDE ANTIBODIES HAVE MORE SUSTAINED CLINICAL RESPONSES THAN PATIENTS NEGATIVE FOR THE MARKER}

C. Wiesenhutter ${ }^{1}$, V. Hayden ${ }^{2} .{ }^{1}$ Coeur d'Alene Arthritis Clinic, Coeur d'Alene, United States of America; ${ }^{2}$ Coeur d'Alene Arthritis Clinic, Coeur d"Alene, United States of America

Background: Treating Rheumatoid Arthritis (RA) patients to target (T2T) has been shown to result in better outcomes in patients with RA [1]. There are now a number of therapeutic options to accomplish this goal, but determining which agent to select for each individual has not been defined.

Objectives: The purpose of this post hoc analysis was to assess if Anticyclic Citrullinated Peptide Antibody (anti-CCP) status effects outcomes following treatment of RA patients with Abatacept.

Methods: Patients at a community based rheumatology clinic undergo disease activity measure assessments on a routine basis as part of the implementation of T2T strategy with ongoing assessments on at least a yearly basis. Over the past 15 years there have been 78 patients initiated on treatment with Abatacept at this clinic. Anti-CCP and Rheumatoid factor status is routinely obtained when patients are first seen in the clinic. A patient was considered to be Anti-CCP positive if the test was $20 \mathrm{u} / \mathrm{ml}$ or greater. As a comparison, the 53 patients in the clinic started on Tofacitinib were also analyzed.

The difference in sustained clinical response rates between seropositive and seronegative patients were determined for these two groups. Sustained clinical response was defined as remaining on treatment for at least three years. Patients who were lost to follow up or who died, while on treatment for less than three years, were not included. Statistical analysis was performed with IBM SPSS V. 25 using chi square tests and logistic regression incorporating pretreatment gender, DAS28CRP, CDAI, rheumatoid factor, Multi-biomarker disease activity score, and a power Doppler joint count.

Results: Fifty anti-CCP positive patients and twenty-two anti-CCP negative patients treated with Abatacept were clinically assessed and results of the post hoc analysis are shown in Table one. Chi square risk estimate 4.61 Clinical sig $\mathrm{p}=0.01$. Logistic regression: Unadjusted Risk ratio $(95 \% \mathrm{Cl}) 4.86(1.54,8.18)$ Clinical sig $p=.01$. Adjusted Risk Ratio $(95 \% \mathrm{Cl}) 4.21$ (1.23, 7.19.) Clinical sig $=0.03$.

Results of the post hoc analysis for patients treated with Tofacitinib are shown in Table two. Chi square risk estimate 1.75 (not clinically significant.) Unadjusted Risk 1.70 (not clinically significant). Adjusted Risk 1.42 (not clinically significant).

\begin{tabular}{|c|c|c|c|c|c|}
\hline & & & Responder & & Total \\
\hline & & & yes & no & \\
\hline \multirow[t]{4}{*}{$\mathrm{CCP}$} & ccp positive & Count & 29 & 21 & 50 \\
\hline & & $\%$ within $\mathrm{CCP}$ & $58.0 \%$ & $42.0 \%$ & $100.0 \%$ \\
\hline & ccp negative & Count & 5 & 17 & 22 \\
\hline & & $\%$ within $\mathrm{CCP}$ & $22.7 \%$ & $77.3 \%$ & $100.0 \%$ \\
\hline \multirow[t]{2}{*}{ Total } & & Count & 34 & 38 & 72 \\
\hline & & $\%$ within $\mathrm{CCP}$ & $47.2 \%$ & $52.8 \%$ & $100.0 \%$ \\
\hline
\end{tabular}

Conclusion: Rheumatoid Arthritis patients who are Anti-CCP positive and who are treated with Abatacept in a community rheumatology clinic have a significantly greater number of sustained clinical responses than patients who are Anti-CCP negative.

\begin{tabular}{|c|c|c|c|c|c|}
\hline & & & \multicolumn{2}{|c|}{ Responder } & \multirow[t]{2}{*}{ Total } \\
\hline & & & yes & no & \\
\hline \multirow[t]{4}{*}{$\mathrm{CCP}$} & ccp positive & Count & 21 & 8 & 29 \\
\hline & & $\%$ within $\mathrm{CCP}$ & $72.4 \%$ & $27.6 \%$ & $100.0 \%$ \\
\hline & ccp negative & Count & 9 & 6 & 15 \\
\hline & & $\%$ within $\mathrm{CCP}$ & $60.0 \%$ & $40.0 \%$ & $100.0 \%$ \\
\hline \multirow[t]{2}{*}{ Total } & & Count & 30 & 14 & 44 \\
\hline & & $\%$ within CCP & $68.2 \%$ & $31.8 \%$ & $100.0 \%$ \\
\hline
\end{tabular}

This difference for patients treated with Tofacitinib was not clinically significant in this clinic, though a higher percentage of Anti-CCP positive patients treated with Tofacitinib responded ( $72 \%$ vs $60 \%$ ).

Anti-CCP positivity could be used as a clinical marker to select patients with rheumatoid arthritis to be treated with Abatacept.

References:

[1] Smolen, J. et al. Ann. Rheum Dis 2010:69;638-643

Disclosure of Interests: None declared

DOI: 10.1136/annrheumdis-2020-eular.2223

\section{\begin{tabular}{|l|l}
\hline AB0232 PAIN SCORE WITH VISUAL ANALOG SCALE OF \\
\hline
\end{tabular} 3OMM OR MORE IS A RISK FACTOR OF WORSENING CLINICAL DISEASE ACTIVITY INDEX (CDAI) AT THREE MONTHS AFTER ATTAINING CDAI REMISSION IN PATIENT WITH RHEUMATOID ARTHRITIS}

I. Yoshii ${ }^{1}{ }^{1}$ Yoshii Hospital, Rheumatology and Musculoskeletal Medicineo, Shimanto City, Japan

Background: In treating with rheumatoid arthritis (RA), it is needless to say essential treatment goal with first priority. On the other hand, patient's pain influences on clinical indices deeply, however, pain score is not been regarded as most important despite that correlates with patient reported outcome.

Objectives: Clinical significance of remnant pain score for clinical outcome although attaining remission in clinical disease activity index (CDAI) statistically.

Methods: RA patient who have attained remission with CDAl were picked up. These patients were divided into two groups whether CDAl at three month after the first CDAI remission attained; namely CDAI-R or CDAI-F. Background data such as sex, age at onset, age, anti-cyclic citrullinated polypeptide antibodies (ACPA), rheumatoid factor (RF), Sharp/van der Heijde Score (SHS), clinical disease activity score (CDAI), C-reactive protein (CRP), modified Health Assessment Questionnaire score ( $\mathrm{mHAQ}$ ), and pain score with visual analog scale (PS-VAS) at first consultation, time span from the first consultation to first CDAl remission were compared between the two groups using Mann-Whitney U-test. CDAI, CRP, mHAQ, PS-VAS, and QOL value calculated from EuroQOL-5 dimension questionnaire $(E Q-5 D)$ at the time of CDAl were also statistically compared with Mann-Whitney U-test. Parameters that demonstrated statistical significance within $5 \%$ were picked up, and odds ratio for CDAI remission were calculated with binary logistic regression analysis. Moreover, parameters that demonstrated statistical significance with $p$-value within $5 \%$ were evaluated with receiver's observational characteristics $(\mathrm{ROC})$ analysis, and cut-off index (COI) was calculated

Results: A total of 907 patients with 594 CDAI-R and $313 \mathrm{CAI}-\mathrm{F}$ were recruied. Demographic characteristics of the two groups were shown in Table 1. SHS at first consultation and time span from first consultation to CDAl remission attained demonstrated significantly less in the CDAI-R than the CDAI-F group, while the other parameters demonstrated no significant difference. CRP, CDAI, $\mathrm{mHAQ}, \mathrm{PS}-\mathrm{VAS}$, and QOL at CDAI remission demonstrated significant difference between the CDAI-R and CDAI-F groups. With binary logistic regression analysis, CRP, CDAl, and PS-VAS demonstrated significant regression for CDAI-R with $1.68,0.71$, and 0.78 in odds ratio, respectively. COI for CDAI remission was $0.4,1.0$, and 30 for CRP $\left(p=2.4 \times 10^{-4}\right)$, CDAI $\left(p=3.0 \times 10^{-32}\right)$, and PS-VAS $(p=2.4$ x $10^{-4}$ ), respectively.

Conclusion: PS-VAS at the moment of CDAI remission is suggested to be predictive factor for sustaining CDAI remission at three months thereafter as well as CRP value and the CDAI score. 
Table 1. Demographic characteristics of the two groups

\begin{tabular}{lccc}
\hline & CDAI-R & CDAI-F & p-value \\
\hline cases & 594 & 313 & \\
sex & $430(72.4 \%)$ & $313(80.2 \%)$ & $5.7 \times 10^{-2}$ \\
age at onset & 62.2 & 60.9 & $1.8 \times 10^{-1}$ \\
age at FC & 65.8 & 65.1 & $4.7 \times 10^{-1}$ \\
ACPA at FC & $209.3(83.9 \%)$ & $227.9(86.7 \%)$ & $7.2 \times 10^{-2}$ \\
RF at FC & $83.8(92.2 \%)$ & $92.3(86.1 \%)$ & $5.6 \times 10^{-2}$ \\
SHS at FC & 41.9 & 66.9 & $6.0 \times 19^{-5}$ \\
CDAI at FC & 10.7 & 11.1 & $5.5 \times 10^{-2}$ \\
CRP at FC & 1.3 & 1.6 & $1.2 \times 10^{-1}$ \\
mHAQ at FC & 0.439 & 0.475 & $3.2 \times 10^{-1}$ \\
PS-VAS at FC & 32.6 & 34.9 & $2.1 \times 10^{-1}$ \\
time span & 3.7 & 4.5 & $6.4 \times 10^{-4}$ \\
\hline
\end{tabular}

Abbreviations: FC, first consultation; ACPA, anti-cyclic citrullinated polypeptide-antibodies; RF, rheumatoid factor; SHS, Sharp/van der eijde Score; CDAl, clinical disease activity index; CRP, C-reacive protein; mHAQ, modified Health Assessment Questionnaire; PS-VAS, pain score with visual analog scale; time span, time span from FC to date first CDAl remission attained.

Disclosure of Interests: None declared

DOI: 10.1136/annrheumdis-2020-eular.1916

\section{$\mathrm{AB} 0233$ \\ ATTAINING CDAI REMISSION IS THE FIRST GATEWAY TO ATTAIN BOOLEAN REMISSION}

I. Yoshii ${ }^{1} .{ }^{1}$ Yoshii Hospital, Rheumatology and Musculoskeletal Medicine, Shimanto-City, Japan

Background: Boolean remission is most stringent but most comparable remission status for the patient with rheumatoid arthritis (RA). Clinical remission evaluated with clinical disease activity index (CDAI) is also one of the most popular index for evaluation of RA treatment. These two criteria often overlap, but some are split.

Objectives: Clinical significance of attaining CDAI remission before attaining Boolean remission was investigated.

Methods: Patient with RA were treated in the institute since August 2010 under treat to target (T2T) strategy. In accordance with T2T, RA patients were monitored from the first consultation with parameter such as tenderness joint count (TJC), swollen joint count (SJC), patient's global assessment (PGA), evaluator's global assessment (EGA), C-reactive protein (CRP), modified Health Assessment Questionnaire (mHAQ), pain scale with visual analog scale (PS-VAS), and EuroQOL 5-dimension (EQ-5D). CDAl and Boolean are also evaluated at the same time. Radiographs of bilateral hands and feet are taken once a year from the first consultation, and the Sharp/van der Heijde Score (SHS) is measured.

In patients, a group who attained CDAI remission prior to attaining Boolean remission (CDAI-R), a group who could not attain CDAI remission previously than attaining Boolean remission (CDAI-F), and a group who could not attain Boolean remission despite attaining CDAI remission (Boolean-F) were picked up and divided according to change of disease activity. Among these three groups, mean age, sex, education level, job style, anti-cyclic citrullinated polypeptide antibodies (ACPA), rheumatoid factor (RF), the CDAl score, the HAQ score, PS-VAS and quality of life index (QOL) calculated from EQ-5D were compared with each other using Mann-Whitney U-test. Boolean remission attaining rate whether CDAI remission attained was compared with chi square test.

Results: Patient group configured with 255 of CDAI-R, 160 of CDAI-F, and 28 of Boolean-F. Patient who could not attain none of CDAI nor Boolean remission counted 175. In background factors at baseline, mean age, the HAQ score, and SHS of the Boolean-F were significantly older than the other groups. In the two groups of CDAI-R and CDAI-F, 28-joints disease activity score with C-reactive protein (DAS28-CRP), CDAI and PS-VAS in the CDAI-R were significantly lower than in the CDAI-F, similarly, DAS28-CRP, the CDAl score, the HAQ score, $P S-V A S$ and QOL after Boolean remission attain were significantly higher in the CDAI-F than the CDAI-R. Sensitivity of Boolean remission when attaining CDAI remission previously before Boolean remission is $93.4 \%$, and specificity was $52.2 \%\left(p<1.0 \times 10^{-30}\right)$.

Conclusion: Attaining CDAI remission previously is extremely important, both for attaining Boolean remission and more stable clinical course after attaining Boolean remission. CDAI remission could be the first gateway to send sustainable QOL course.

Disclosure of Interests: None declared

DOI: 10.1136/annrheumdis-2020-eular.1550

\section{Rheumatoid arthritis - comorbidity and clinical aspects}

\section{$\mathrm{AB} 0234$ \\ ASSOCIATION OF HOMOCYSTEINE LEVEL AND CAROTID INTIMA-MEDIA THICKNESS IN PATIENTS WITH RHEUMATOID ARTHRITIS}

D. Anghel ${ }^{1}$, L. Otlocan ${ }^{2}$, R. Bursuc ${ }^{1}$, E. Busuioc ${ }^{1}$, A. Manolache ${ }^{1}$, F. C. Plesa ${ }^{1}$, V. Smedescu ${ }^{1}$, M. M. Negru ${ }^{3}$, C. Jurcuț ${ }^{1} .{ }^{1}$ Central Military Emergency University Hospital "Carol Davila", Bucharest, Romania; "Sfanta Maria"Clinic, Bucharest, Romania; “"Sfanta Maria”Clinical Hospital, Bucharest, Romania

Background: Homocysteine (Hcy) has been implicated in atherogenesis. High homocysteine level can predict cardiovascular events, including death. Atherosclerosis has a high incidence in patients with Rheumatoid Arthritis (RA).

Objectives: The aim of this study is to evaluate the relationship between serum homocysteine levels and carotid atherosclerosis in patients with RA and antiTNF therapy.

Methods: Our study included 80 RA patients divided into two groups: 45 patients were with anti-TNF-alpha therapy (Adalimumab, Infliximab, Etanercept) and 35 RA patients with disease-modifying antirheumatic drugs (DMARDs). The patients were diagnosed with RA used ACR/EULAR 2010 Classification Criteria. We measured carotid intima-media thickness (CIMT) using high-resolution Doppler ultrasonog raphy at baseline and then at 12 months. CIMT above $0.9 \mathrm{~mm}$ is an atherosclerosis marker. We considered high levels of homocysteine in the serum above 15 $\mu \mathrm{mol} / \mathrm{L}$. All patients had treatment with hypolipemiant drugs and antiplatelet agents during the 12 months. Other parameters were analyzed at baseline and after 12 months: age, lipid profile (HDL, LDL, and cholesterol), ESR and disease activity score (DAS28<2.6 means remission; DAS28=2.6-3.2 means low disease activity, DAS28=3.2-5.1 means moderate disease activity; DAS28 $>5.1$ high disease activity). Results: 45 patients received anti-TNF-alpha therapy (mean age $45.50 \pm 9.69$ years) and 35 RA patients had treatment with DMARDs (mean age $48.3 \pm 8.9$ years). High Hcy levels were found on $34 \%$ patients in DMARDs group and $21 \%$ patients in anti-TNF group. After 12 months of treatment, patients with high levels of Hcy and anti-TNF therapy had a significant decrease in CIMT. In patients with low Hcy level the decrease in CIMT was insignificantly statistic. In DMARDs group atherosclerotic plaque was detected to 26 patients $(74.29 \%)$ and 21 (46.66\%) patients were detected into anti-TNF group. After 12 months CIMT was significantly higher in DMARDs group and the difference was statistically significant compared to baseline and to anti-TNF group $(p=0.0002)$. High DAS28 score was associated with increased CIMT and hyperhomocysteinemia in both groups $(p=0.0001)$. Conclusion: Increased Hcy levels were associated with increased CIMT values in both groups. In RA patients with anti-TNF therapy and high Hcy levels, reduction of CIMT was statistically higher than in patients with DMARDs treatment.

Disclosure of Interests: None declared

DOI: 10.1136/annrheumdis-2020-eular.2690

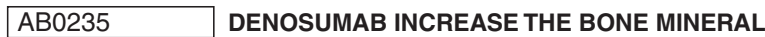 DENSITY REGARDLESS OF DISEASE ACTIVITY, THE BIOLOGICAL DISEASE-MODIFYING ANTIRHEUMATIC DRUGS, THE CONCOMITANT TYPE OF VITAMIN D, AND PRETREATMENT OF OSTEOPOROSIS IN PATIENTS WITH RHEUMATOID ARTHRITIS.}

S. Anno ${ }^{1,2}$, T. Okano ${ }^{2}$, K. Inui ${ }^{2}$, T. Koike ${ }^{3,4}$, H. Nakamura ${ }^{2}{ }^{1}$ Osaka Social Medical Center, Department of Orthopaedic Surgery, Osaka, Japan; ${ }^{2}$ Osaka City University Medical School, Department of Orthopaedic Surgery, Osaka, Japan; ${ }^{3}$ Osaka City University Medical School, Center for Senile Degenerative Disorders (CSDD), Osaka, Japan; ${ }^{4}$ Search Institute for Bone and Arthritis Disease (SINBAD), Shirahama, Japan

Background: Osteoporosis is one of the major comorbidities in patients with rheumatoid arthritis (RA). There are a lot of evidence that denosumab increase bone mineral density (BMD) in patients with osteoporosis. However, there are few reports investigated the influence of denosumab in patients with RA.

Objectives: We evaluated the BMD change in patients with RA treated denosumab and assessed the effect of various factors, such as disease activity, biological disease-modifying anti-rheumatic drugs (bDMARDs) use, concomitant medications of osteoporosis and pretreatment of osteoporosis.

Methods: This study included 140 consecutive RA patients (135 female, mean age was $70.6 \pm 8.6$ years) who fullfilled the criteria of osteoporosis and treated with denosumab. BMD at the lumbar spine, proximal femoral and femoral neck were evaluated by dual energy X-ray absorptiometry at baseline and one year after treatment. We evaluated the influence of disease activity, bDMARDs use, 